\section{Mapping of Quantitative Trait Loci Involved in Ornamental Traits in Alstroemeria}

\author{
Tae-Ho Han, Herman J. van Eck ${ }^{1}$, Marjo J. De Jeu, and Evert Jacobsen \\ Laboratory of Plant Breeding, The Graduate School Experimental Plant \\ Science, Wageningen University and Research Centre, P.O. Box 386, NL-6700 \\ AJ Wageningen, The Netherlands
}

Additional index words. Alstroemeria, ALFP markers, outbreeder, QTL mapping

\begin{abstract}
An $\mathrm{F}_{1}$ population, derived from an intraspecific cross between two Alstroemeria aurea accessions, was used to map quantitative trait loci (QTL) involved in ornamental and morphological characteristics. One QTL for leaf length was mapped on linkage group three of both parents near marker E+ACCT/M+CGCA-I165 explaining 20\% and $\mathbf{1 4 . 8 \%}$ phenotypic variation. Two putative QTL were detected on leaf width on A002-3 and A002-6. One QTL and three putative QTL, involved in the leaf length/width ratio were identified accounting for $\mathbf{4 6 . 7 \%}$ of the phenotypic variance in total. Significant interaction was observed between two QTL, S+AC/M+ACT-I162 and S+AC/M+AGAI465 in a two-way analysis of variance (ANOVA). For the main color of the flower one QTL and putative QTL accounted for up to $60 \%$ of phenotypic variance suggesting simple genetic control of flower color. A two-way ANOVA of these QTL suggested an epistatic interaction. A QTL was detected for color of the inner side of outer lateral tepal with $26.5 \%$ of the phenotypic variance explained. This QTL was also associated with main color of the flower just below the $95 \%$ threshold value. Two QTL were detected with the Kruskal-Wallis test for the tip color of inner lateral tepal near QTL for other flower color traits. Consequently flower color traits were significantly correlated. A QTL and a putative QTL for the flower size was mapped near marker E+ACCG/ M+CGCT-I193 and E+ACCG/M+CGCG-197, respectively. One putative QTL was detected for the stripe width of the inner lateral tepal.
\end{abstract}

Alstroemeria is a cut flower, mainly originating from Chile and Brazil. It has very colorful flowers with a long vase life, and it is, therefore, a commercially important ornamental crop in the Netherlands. As in all ornamentals, the morphological traits of flower and leaf are very important characteristics in Alstroemeria breeding programs. Alstroemeria accessions and cultivars are noninbred and are maintained vegetatively. The large genome size of Alstroemeria aurea of $4 \mathrm{C}=107$ pg (Buitendijk et al., 1997), which equals 25,000 Mbase, provides a limitation to several molecular marker techniques. In an earlier study, we adapted the AFLP technique for Alstroemeria by increasing the number of selective nucleotides added to the core primers. This resulted in accurate and reproducible fingerprints despite the complex template (Han et al., 1999). By using a rare cutter (8-cutter: Sse8387I) for the preparation of primary template, a more simple template could be obtained, and reproducible fingerprints could be generated with the usual number of selective nucleotides (Han et al., 2001). Similarly, the AFLP technique was also applied in onion

Received for publication 6 Feb. 2001. Accepted for publication 5 Sept. 2001. We thank Cornelia Maria Kreike for critical reading of the manuscript. The $A$. aurea accessions A002 and A003 were kindly provided by the breeding company Wülffinghof BV, The Netherlands.

${ }^{1}$ To whom reprint requests should be addressed. E-mail address: herman.vaneck@pv.dpw.wag-ur.nl tal characteristics of $A$. aurea were available, guidelines.

${ }^{2}$ Trait analyzed only by Kruskal-Wallis test. marker-assisted selection could be performed in seedling stages and used to eliminate undesirable individuals from progeny populations. Linkage relationships between molecular markers and morphological traits have been studied in many crop plants such as potato (Solanum tuberosum L.) (Van Eck et al., 1993; Van Eck et al., 1994a), wheat (Triticum sp.) (Kato et al., 1999), sunflower (Helianthus annus L.) (Gentzbittel et al., 1999), tomato (Lycoperscion esculentum Mill.) (Grandillo et al., 1999), barley (Hordeum vulgare L.) (Zhu et al., 1999), and cotton (Gossypium sp.) (Jiang et al., 2000). In ornamentals, flower traits such as petal number and flower color have recently been mapped for the first time in rose by using RAPD and AFLP markers (Debener and Mattiesch, 1999). No similar study has been reported in Alstroemeria before this paper.

The recent construction of the first molecular linkage map in A. aurea (accession A002 $x$ accession A003) using AFLP markers (Han et al., 2001) facilitated the first quantitative trait loci (QTL) analysis in A. aurea. We present data on 14 traits that are important in ornamentals. Subsequently, the inheritance of these traits has been selected by QTL analysis, to identify genetic loci responsible for the morphological variation in $A$. aurea accessions.

\section{Materials and Methods}

Plant material. A population of $134 \mathrm{~F}_{1}$ individuals was obtained from an intraspecific cross of the A. aurea accessions; A002 $\times$ A003 (Han et al., 2001). The genotypes A002 and A003, which are commonly used progenitor lines from breeding companies, showed distinctive morphological differences such as in flower color; red and yellow respectively. Parents and the $F_{1}$ descendants were grown under the same greenhouse conditions.

Phenotypic analysis. The 134 individual $\mathrm{F}_{1}$ plants were scored for 14 morphological and ornamental traits (Table 1). The majority of these traits were chosen on the basis of the Union internationale pour la protection des obtentions vegetales (UPOV) list of cultivar descriptors (UPOV, 1987). UPOV character-

Table 1. List of morphological, ornamental, or both characters studied, the parental trait values and the trait number according to the Union internationale pour la protection des obtentions vegetales (UPOV)

\begin{tabular}{lccc}
\hline $\begin{array}{l}\text { Trait } \\
\text { code }\end{array}$ & $\begin{array}{c}\text { Trait } \\
\text { name }\end{array}$ & $\begin{array}{c}\text { Trait values of } \\
\text { genotypes A002; A003 }\end{array}$ & $\begin{array}{c}\text { UPOV } \\
\text { character no. }\end{array}$ \\
\hline L1 & Leaf length & $9.99 ; 12.84 \mathrm{~cm}$ & 4 \\
L2 & Leaf width & $1.77 ; 1.65 \mathrm{~cm}$ & 5 \\
L3 & Ratio of leaf length and width & $5.67 ; 7.87$ & 6 \\
L4 & Length of leaf petiole & $2.05 ; 2.26 \mathrm{~cm}$ & 7 \\
C1 & Main color of complete flowers & Red; Yellow & 11 \\
C2 & Color of inner side of outer lateral tepal & Red; Yellow & 16 \\
C3 & Tip color of inner lateral tepal & Red; Yellow & 20 y \\
F1 & Flower size & $3.92 ; 4.52 \mathrm{~cm}$ & 12 \\
F2 & Flower openness & Open; Close & 13 \\
F3 & Number of flowering stems & Not determined for the parents & --- \\
F4 & First date of flowering & 4 June; 10 April & --- \\
F5 & Last date of flowering & 28 June; 15 June & --- \\
F6 & Flowering period in days & $24 \mathrm{~d} ; 66 \mathrm{~d}$ & --- \\
S1 & Stripe width of inner lateral tepal & $0.83 ; 0.73 \mathrm{~mm}$ & 22 \\
\hline
\end{tabular}

${ }^{\text {y} M o d i f i e d ~ U P O V ~ c h a r a c t e r . ~ N o t ~ m i d d l e ~ z o n e, ~ b u t ~ t i p ~ c o l o r ~ o f ~ t h e ~ i n n e r ~ l a t e r a l ~ t e p a l ~ w a s ~ s t u d i e d . ~}$ 
istics that did not segregate were not studied, and characters that did segregate were included, although they were not part of the UPOV list. Obviously, the UPOV list includes traits that are very stable, highly heritable and not much affected by environmental factors. The genotypes that did not flower were treated as missing values unless it was specified. From each plant, five mature leaves were taken from five individual vegetative stems to measure length (UPOV characteristic No. 4) and width (UPOV characteristic No. 5) of the leaves, to calculate the length/width ratio of the leaves (UPOV characteristic No. 6) and to measure the length of the petiole (UPOV characteristic No. 7). Flower color was dissected into three traits: 1) the main color of complete flowers (UPOV characteristic No. 11); 2) the color of the inner side of the outer lateral tepal (UPOV characteristic No. 16); and 3) the color of the tip of the inner lateral tepal. Each of these traits was scored into five classes; yellow (A003), yellow-orange, orange, orangered, and red (A002). Flower size was recorded by measurements of the length of the outer lateral tepal (UPOV characteristic No. 14). Flower openness was measured into three classes, open (A002), intermediate, and close (A003) (UPOV characteristic No. 13). This trait was defined as "open" when the distance between the tips of the tepals was large, "close", when the tepals were compact together without any space. Productivity was measured by counting the number of flowering stems. Plants that did not flower were scored as missing value. The flowering period was measured in terms of date of appearance of the first and last flowering stem, and flowering period in days. Flowering period in days was transformed by square root to achieve the normal distribution. The width of the stripes shown on the inner side of the inner lateral tepal was measured in two different flowers using a calliper rule when the flower was mature (UPOV characteristic No. 22).

Genotyping and data analysis. AFLP segregation data and marker order on A. aurea linkage maps, used in this study for QTL identification, have been described previously (Han et al., 2001). QTL detection was based on separate parental data sets and nonintegrated maps of A002 and A003. Each of the parental data sets does include some allelic bridge markers, which segregate in a 3:1 ratio. However, the position of these 3:1 markers on the A002 and A003 linkage groups cannot be accurately determined (Maliepaard et al., 1997). As a consequence, the marker order on the integrated map will be also highly ambiguous, even if the marker order within each parental map would be fixed. Moreover, not all linkage groups could be integrated with a homologous group from the other parent. A more accurate integration of the A002 and A003 linkage groups would require at least codominant markers, and preferably allowing full classification such as SSR or RFLP markers. These are not available in Alstroemeria.

Average trait values and correlation coefficients between traits were calculated to de- tect putative relationships between various characters. Correlations were tested using the parameter free Spearman's rank correlation test (two sided, $P>0.01$ ).

Two analytical approaches were used to identify putative QTL and to estimate their phenotypic effects: the Kruskal-Wallis test (Kruglyak and Lander, 1995) and interval mapping (Lander and Botstein, 1989). For both analyses, the software package MapQTL ${ }^{\circledR}$ Version 4.0 (Van Ooijen, 2000) was used. QTL maps were drawn by using MapChart program that was kindly provided from Voorrips (2000). Both interval mapping and Kruskal-Wallis test was used to analyze the metric traits, where the trait values represented a normal distribution. Trait values that represent an ordinal distribution, such as the five shades of color, ranging from yellow to red, were also analyzed with both interval mapping and Kruskal-Wallis test. Although these traits were ordinal, analysis of variance (ANOVA) with marker class as explanatory factor showed that the residuals, and thus the trait, behaved more or less in accordance with standard ANOVA assumptions. Therefore, the results of QTL analysis on these traits, as if it were truly quantitative, should allow sufficiently reliable conclusions. Flower openness was a discrete trait, and therefore analyzed only by Kruskal-Wallis test.

In order to keep the probability of claiming one false-positive QTL below 5\% in the Alstroemeria genome [ 8 chromosomes, $\approx 305$ 605 centiMorgan map units (cM)], a probability level of $P<0.005$ was chosen for the rank sum test of Kruskal-Wallis, to declare a QTL significant (Van Ooijen, 2000). For interval mapping, a permutation test was used to empirically determine the significance threshold of the LOD score for each linkage group (Churchill and Doerge, 1994). The frequency distribution of the maximum LOD score was determined by 1000 permutations of the quantitative trait data over individuals while the marker data remained fixed. LOD scores that exceeded $99 \%$ (or 95\%) of this distribution were declared significant.

\section{Results}

The distribution for the morphological and ornamental traits in the $\mathrm{F}_{1}$ population is shown in Fig. 1, together with an indication of the parental trait values. These parental trait values are also listed in Table 1. For all traits, except for flower openness, the offspring trait values displayed a continuous distribution, not deviating from normality. None of the traits were correlated (data not shown) except for those cases where correlation was obvious. An obvious correlation should be expected between color of the different parts of the flowers (e.g. C1, C2, and C3 were correlated), and correlation between the different aspects of leaf shape (L1, L2, L3, and L4).

Marker-trait associations are shown in Table 2 and Fig. 2. Interval mapping allowed the detection of three QTL exceeding the 99\% significant threshold obtained by permutation. When allowing a 95\% significant threshold, another 14 QTL were detected, but we prefer to call these putative QTL. The Kruskal-Wallis test allowed the detection of four significant QTL at $P \leq 0.001$, and another four putative QTL at a significance threshold of $P \leq 0.005$.

Leaf traits $Q T L$. The parents of the $\mathrm{F}_{1}$ population showed differences in leaf length and leaf width; A002 was shorter and wider $(9.99 \mathrm{~cm}$ and $1.77 \mathrm{~cm}$ ), whereas A003 was longer and narrow $(12.84 \mathrm{~cm}$ and $1.65 \mathrm{~cm})$ (Table 1). One QTL, involved in leaf length (L1) was mapped on linkage group three of both parent A002-3 and A003-3 near marker $\mathrm{E}+\mathrm{ACCT} / \mathrm{M}+\mathrm{CGCA}-\mathrm{I} 165$ which also segregates from both parents (Table 2 and Fig. 2). At the QTL peak position, near marker E+ACCT/M+CGCA-I165, 20.0\% of the phenotypic variance could be explained using markers from linkage group A002-3. The same estimate but based on markers from linkage group A003-3 explained 14.8\% of the phenotypic variance. A marker that would allow full classification at this locus is desired to allow a more accurate estimate. This QTL had the effect to increase the leaf length when the marker alleles were homozygous absent.

Two putative QTL, near marker E+ACCT/ $\mathrm{M}+\mathrm{CGCA}-\mathrm{I} 165$ and S+AT/M+AGA-164, were detected on leaf width (L2) on two different linkage groups, A002-3 and A002-6. These putative QTL explained $13.8 \%$ and $24.7 \%$ of the phenotypic variance, respectively. For both putative QTL, the leaf width was smaller when the marker band was present.

The ratio of the leaf length and width (L3) was used to describe the shape of leaf. Three putative QTL, involved in the leaf length : width ratio were identified with interval mapping and one significant QTL, exceeding the $P \leq 0.001$ significance threshold of the Kruskal-Wallis test, was identified on linkage group 5 of parent A003. This Kruskal-Wallis detected QTL was not observed by interval mapping because it did not reach the $95 \%$ significance threshold. The three putative QTLs obtained by interval mapping were all positioned on linkage group 4; two QTL peaks were observed in parent A002, and a QTL on the homologous linkage groups of the other parent (Table 2). All four QTL together accounted in total for $46.7 \%$ of the phenotypic variance. No interaction was detected between the QTL of linkage groups 4 and 5 (data not shown). However, significant interaction was observed between markers $\mathrm{S}+\mathrm{AC} / \mathrm{M}+\mathrm{ACT}$ I162 and S+AC/M+AGA-I465 in a two-way ANOVA (data not shown). Full classification of this QTL locus by 1:1:1:1 segregating markers (or a well integrated map of A002 and A003) is required to describe this QTL locus in greater detail.

For the length of the leaf petiole (L4) one putative QTL, near marker E+ACCC/ M+CGAG-900 was detected on A002-1 only by Kruskal-Wallis test with $8.8 \%$ of the phenotypic variance. A short leaf petiole was associated with the presence of the band.

Flower traits QTL. The parents, $\mathrm{A} 002$ and A003, differed in their main flower color, red and yellow, respectively (Table 1). A continuous range of different shades of colors be- 

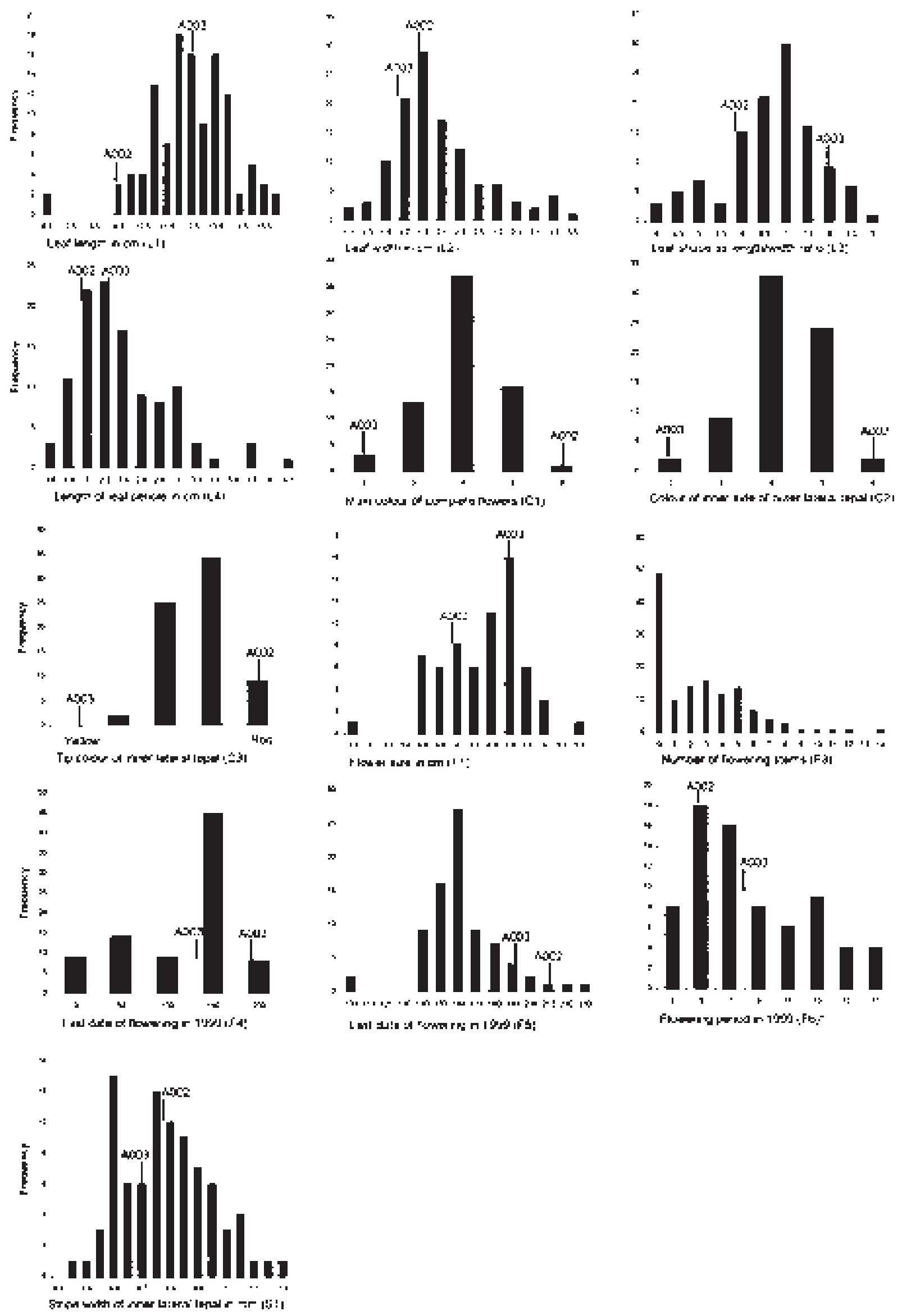

Fig. 1. Histograms of genotype estimates for traits relating to morphological traits scored on individuals from the segregating population derived from 'A002 $\times$ A003'. Mean parental values are indicated with lines. The phenotypic values are transformed by square root. 
Breeding, Cultivars, Rootstocks, \& Germplasm Resources

Table 2. Position and nearest markers of QTL (bold) and putative QTL detected for either morphological, ornamental, or both traits, as detected by interval mapping or Kruskal-Wallis test.

\begin{tabular}{|c|c|c|c|c|c|c|c|}
\hline \multicolumn{8}{|c|}{ Interval mapping } \\
\hline $\begin{array}{l}\text { Trait } \\
\text { code }\end{array}$ & $\begin{array}{l}\text { Linkage } \\
\text { group }^{2}\end{array}$ & $\begin{array}{c}\text { LOD peak } \\
\text { position }(\mathrm{cM})\end{array}$ & $\begin{array}{l}\text { Nearest } \\
\text { marker }\end{array}$ & $\begin{array}{c}R^{2} \\
(\%)^{\mathrm{y}}\end{array}$ & LOD & $95 \%{ }^{x}$ & $99 \%{ }^{\mathrm{w}}$ \\
\hline$\overline{\mathrm{L} 1}$ & A002-3 & 28.8 & E+ACCT/M+CGCA-I165 & 20.0 & 4.81 & 2.9 & 3.7 \\
\hline L1 & A003-3 & 39.0 & $\mathrm{E}+\mathrm{ACCT} / \mathrm{M}+\mathrm{CGCA}-\mathrm{I} 165$ & 14.8 & 3.63 & 3.0 & 3.7 \\
\hline L2 & A002-3 & 28.8 & $\mathrm{E}+\mathrm{ACCT} / \mathrm{M}+\mathrm{CGCA}-\mathrm{I} 165$ & 13.8 & 3.42 & 3.5 & 4.9 \\
\hline L2 & A002-6 & 37.7 & S+AT/M+AGA-164 & 24.7 & 3.57 & 3.0 & 4.4 \\
\hline L3 & $\mathrm{A} 002-4$ & 1.3 & $\mathrm{~S}+\mathrm{AG} / \mathrm{M}+\mathrm{ATT}-113$ & 12.8 & 2.59 & 2.4 & 3.2 \\
\hline L3 & A002-4 & 15.9 & $\mathrm{~S}+\mathrm{AC} / \mathrm{M}+\mathrm{ACT}-\mathrm{I} 162$ & 11.2 & 2.79 & 2.4 & 3.2 \\
\hline L3 & A003-4 & 39.7 & $\mathrm{~S}+\mathrm{AC} / \mathrm{M}+\mathrm{AGA}-\mathrm{I} 465$ & 13.8 & 3.45 & 3.3 & 3.8 \\
\hline $\mathrm{C} 1$ & A003-1 & 47.1 & E+AATC/M+CGCG-I168 & 27.8 & 4.18 & 3.4 & 4.1 \\
\hline $\mathrm{C} 1$ & $\mathrm{~A} 003-2$ & 62.6 & $\mathrm{E}+\mathrm{ACCA} / \mathrm{M}+\mathrm{CGAA}-150$ & 32.2 & 3.57 & 3.1 & 4.5 \\
\hline $\mathrm{C} 2$ & A003-9 & 26.5 & E+AATT/M+CGAT-222 & 26.5 & 2.70 & 2.7 & 3.7 \\
\hline $\mathrm{C} 3$ & A003-1 & 47.1 & E+AATC/M+CGCG-I168 & 24.5 & 3.35 & 3.3 & 4.1 \\
\hline F1 & A002-5 & 9.1 & E+ACCG/M+CGCT-I193 & 31.6 & 3.85 & 3.0 & 3.8 \\
\hline F1 & A003-2 & 38.1 & E+ACCG/M+CGCG-197 & 31.9 & 3.06 & 3.0 & 3.8 \\
\hline S1 & A002-2 & 47.1 & $\mathrm{~S}+\mathrm{AG} / \mathrm{M}+\mathrm{ATT}-\mathrm{I} 79$ & 15.5 & 2.97 & 2.8 & 3.6 \\
\hline \multicolumn{8}{|c|}{ Kruskal-Wallis test } \\
\hline
\end{tabular}

\begin{tabular}{lcccrr}
\hline $\begin{array}{l}\text { Trait } \\
\text { code }\end{array}$ & $\begin{array}{c}\text { Linkage } \\
\text { group }\end{array}$ & $\begin{array}{c}\text { Marker } \\
\text { position }(\mathrm{cM})\end{array}$ & Marker & $\mathrm{K}^{\mathrm{v}}$ & $P$ value \\
\hline L3 & A003-5 & 39.6 & E+AATC/M+CGCG-123 & $\mathbf{1 1 . 5 7 0}$ & 0.001 \\
L4 & A002-1 & 6.9 & E+ACCC/M+CGAG-900 & 10.414 & 0.005 \\
C3 & A002-1 & 42.5 & E+ACCC/M+CGCG-93 & $\mathbf{1 2 . 4 5 5}$ & 0.0005 \\
C3 & A003-9 & 26.0 & E+AATT/M+CGAT-248 & $\mathbf{1 0 . 9 8 9}$ & 0.001 \\
S1 & A003-4 & 54.8 & E+AATT/M+CGCA-65 & 10.330 & 0.005 \\
F2 & A003-9 & 0.0 & S+AC/M+AGA-53 & 10.514 & 0.005 \\
F4 & A002-7 & 8.9 & E+AATC/M+CGCG-I112 & 8.714 & 0.005 \\
F5 & A003-1 & 58.7 & E+ACCA/M+CGCC-222 & $\mathbf{1 1 . 2 6 5}$ & 0.001 \\
\hline
\end{tabular}

${ }^{2}$ Linkage groups as assigned in Han et al. (submitted)

yercentage of variance explained.

${ }^{x}$ LOD threshold values at $95 \%$ confidence level obtained by permutation test.

wLOD threshold values at $99 \%$ confidence level obtained by permutation test

"Kruskal-Wallis test statistic $(\mathrm{K})$.

tween yellow and red was observed in the offspring. For the main color of the flower (C1) we identified one QTL (exceeding 99\% significance threshold) near markerE+AATC/ M+CGCG-I168 on linkage group A003-1. In addition, one putative QTL (95\% threshold) was identified near marker E+ACCA/ M+CGAA-150, on the linkage group A003-2. The total variance explained by these two QTL sums up to $60 \%$. This suggests that flower color is under simple genetic control, because a major proportion of the phenotypic variance is explained by these two loci. Statistical analysis by a two-way ANOVA of the joint action of these two QTL showed a significant interaction component $(F=5.56, P=$ $0.0221)$ indicating epistatic interaction. This may also explain the discrepancy between the high amounts of explained variance, in contrast with marginally significant LOD scores.

A QTL near marker E+AATT/M+CGAT222 was involved in color of the inner side of outer lateral tepal (C2) with $26.5 \%$ of the phenotypic variance explained. The color of these outer tepals constitutes a major aspect of the main color of the flower. Although there is no overlap between the QTL of the traits C1 and $\mathrm{C} 2$, marker E+AATT/M+CGAT-222 was also associated with main color of the flower at a LOD of 2.65, which is just below the 95\% threshold value $(\mathrm{LOD}=2.9)$.

Two QTL, near marker $\mathrm{E}+\mathrm{ACCC} /$ M+CGCG-93 and E+AATT/M+CGAT-248, were detected with the Kruskal-Wallis test for the tip color of inner lateral tepal (C3). The QTL detected by E+ACCC/M+CGCG-93 on linkage group A002-1 is possibly the same QTL as the one that was detected by interval mapping near marker E+AATC/M+CGCGI168 on linkage group A003-1. This could suggest a single gene that is heterozygous in both parents. The QTL near marker E+AATC/ $\mathrm{M}+\mathrm{CGCG}-\mathrm{I} 168$ on linkage group 1 is involved in two related traits, the main color of complete flowers and tip color of the inner lateral tepal. The QTL, near marker E+AATT/ $\mathrm{M}+\mathrm{CGAT}-248$, for the tip color of the inner lateral tepal was mapped very close to $(0.5$ cM) marker E+AATT/M+CGAT-222, associated with the color of the inner side of the outer lateral tepal. Consequently, flower color traits were significantly correlated (data not shown).

A QTL for the length of the outer lateral tepal, representing flower size (F1), was mapped near marker E+ACCG/M+CGCTI193 on linkage group A002-5 and a putative QTL near marker E+ACCG/M+CGCG-197 on linkage group A003-2. Each QTL explained $32 \%$ of the phenotypic variance (Table 2 ).

Flower openness segregated in the offspring, but the amount of variation in this offspring is rather subtle because the parents differ only slightly. The range of variation is ranging between the values 5 and 7 of the UPOV list, which is typical for Chilean species such as A. aurea. The more cylindrical flower shapes, typical for Brazilian species, should not be expected in this gene pool. In our case, we did not attempt to classify the variation in the offspring in more than three classes: "close," "intermediate," and "open" resembling UPOV value 5, 6, and 7, respectively. A putative QTL, at the end of the linkage group marked by $\mathrm{S}+\mathrm{AC} / \mathrm{M}+\mathrm{AGA}-$ 53, involved in flower openness (F2), was detected on linkage group A003-9 by the Kruskal-Wallis test.

Several aspects of productivity have been studied. Productivity of cut flowers can be represented by the number of flowering stems. Therefore, the number of flowering stems was counted for during the flowering season. However, out of the 134 genotypes 49 did not produce any flowering stem. The trait value of a genotype that did not flower could be indicated with a 'zero' or it could be a missing value. Both options have been tested in QTL analysis and both options resulted in putative QTL. However, the putative QTL observed by interval analysis were not significant with the Kruskal-Wallis test, and vice versa. Moreover, none of these putative QTL was detected for both options. A third option to analyze this trait is to treat it as a binary character: Yes or No flowering. This did not result in the detection of any genetic locus. In view of these contradictory results, we do not feel confident to mention any of the putative QTL.

Another aspect of productivity, which is highly confounded with the number of flowering stems, is the flowering period. When more stems are produced, the period will also be longer. Three aspects of flowering period were studied: 1) first date of flowering, representing earliness of the crop; 2) last date of flowering, representing the joint effect of number of stems and lateness of the crop; and 3) duration of flowering period, which is the interval between the first two traits. A putative QTL for first date of flowering $(\mathrm{F} 4)$ was detected with marker E+AATC/M+CGCG-I112 on A002-7 with $7.7 \%$ of the phenotypic variance explained. A putative QTL marker for last date of flowering (F5) was marker E+ACCA/ M+CGCC-222 on A003-1 with $13.3 \%$ of the phenotypic variance explained. No QTL were detected for duration of flowering period.

Stripe trait QTL. One putative QTL marker $\mathrm{S}+\mathrm{AG} / \mathrm{M}+\mathrm{ATT}-\mathrm{I} 79$ was detected for the stripe width of the inner lateral tepal (S1) on linkage group A002-2. This QTL accounted for 15\% of the phenotypic variance, and wider stripes were associated with the presence of the band.

\section{Discussion}

In the offspring of the cross between Alstroemeria accessions A002 $\times$ A003, a large number of morphologically and/or ornamentally important traits segregated, resulting in highly diverse offspring genotypes. This rich genetic variation was studied by QTL analysis using AFLP markers mapped on separate parental linkage groups of A002 and A003. The range of phenotypes observed in the offspring is described by the frequency distributions of the trait values in Fig. 1, together with the parental trait values. For the morphological traits of the leaves/length, width, shape (length : width ratio) and leaf petiole length, flower size, stripe width, and three aspects of flowering period, phenotypic values have been ob- 


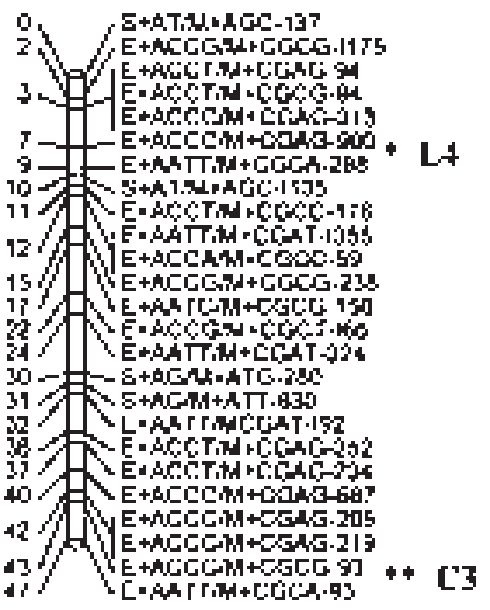

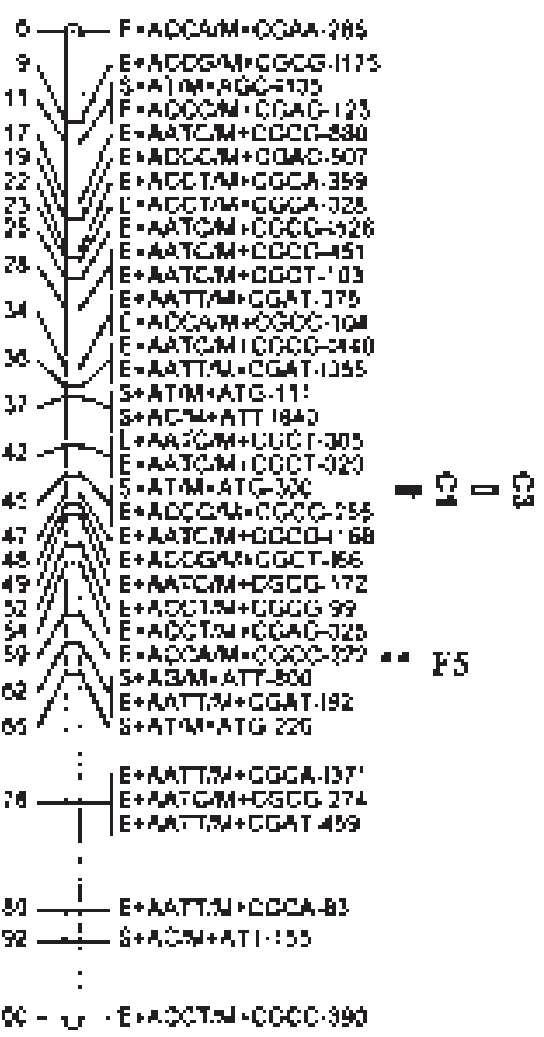

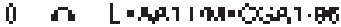

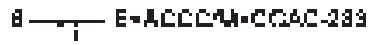

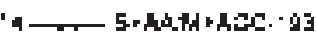

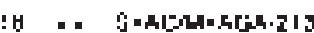

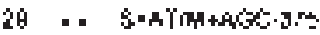

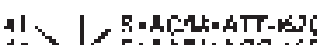

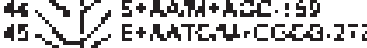
T...

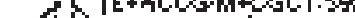

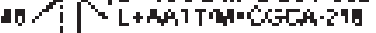
5 C4 [+m
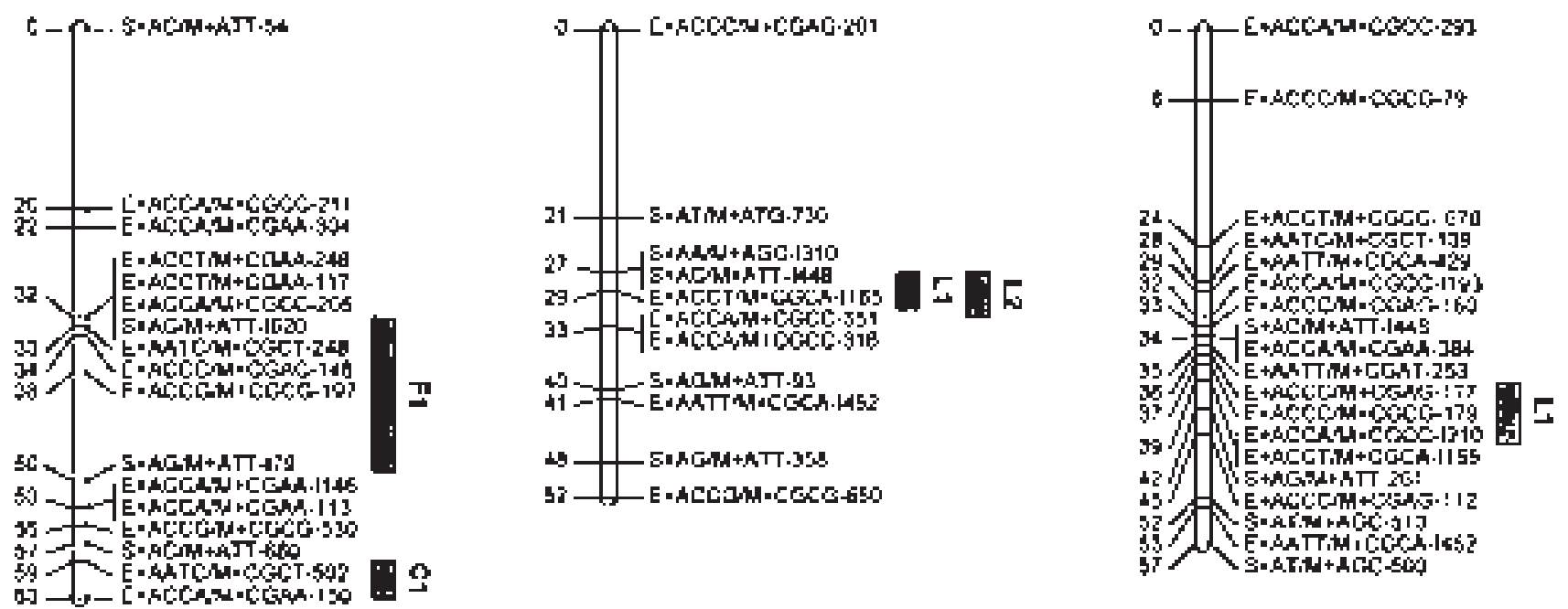

Fig. 2. QTL (solid bars or**) and putative QTL (hatched bars or *) associated with the traits are depicted along the linkage groups of the A. aurea map. QTL detected by interval mapping are indicated by a bar. The length of the bar equals the 1 LOD support interval. QTL detected by Kruskal-Wallis are indicated by one or two asterisk attached to the marker position. (Fig. 2 continues on next page.) 


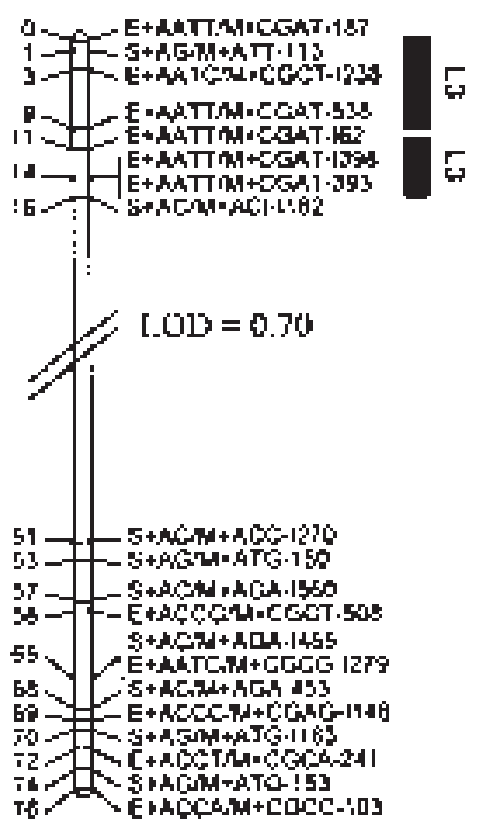

$\sin -5$
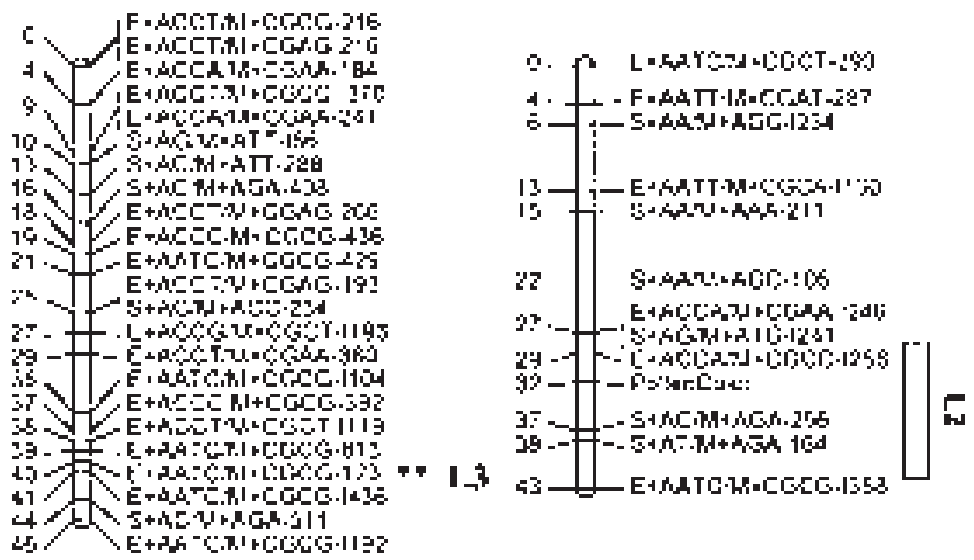

Fig. 2. continued.

A. 50.2.5
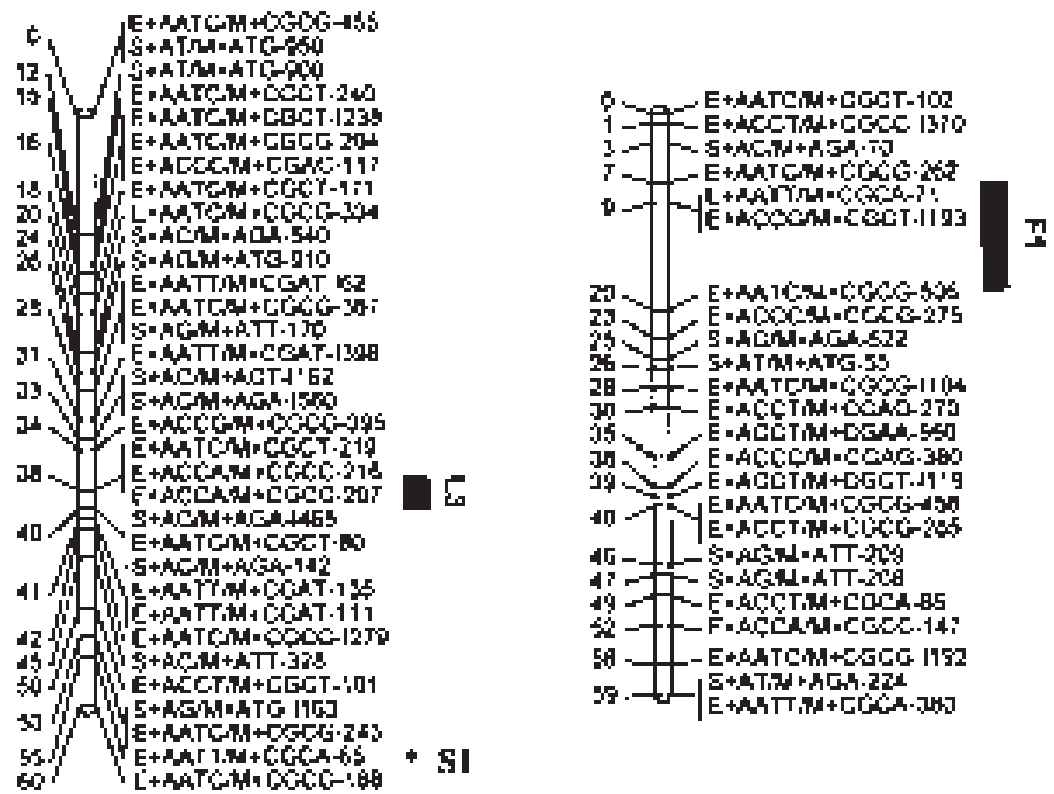

Hobsis

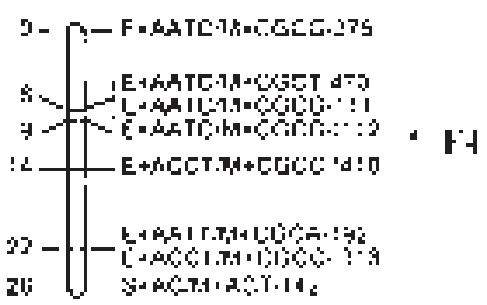

And-3

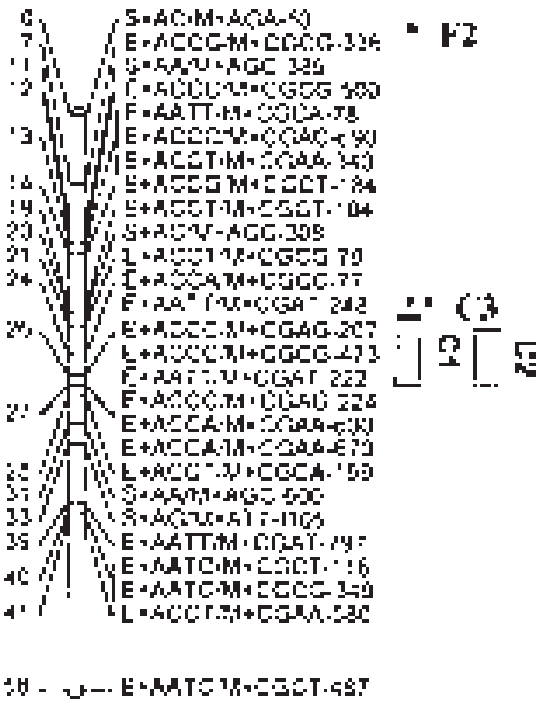

HortScience, Vol. 37(3), June 2002 
served in the progeny that go beyond the parental values. These transgressive segregations are typical for the offspring of noninbred species, and result from heterozygosity in the parents for QTL alleles with positive as well as negative effects. In the case of flower color traits $(\mathrm{C} 1, \mathrm{C} 1$, and $\mathrm{C} 3)$ the parents showed extreme phenotypes, with offspring phenotypes ranging between the parental extremes. Nevertheless, extreme parental phenotypes did not result from fixation (homozygosity) at genes involved in yellow and red flower pigments, because homozygosity at these loci would have resulted in a uniform $F_{1}$. On the contrary, heterozygosity was present both in parent A002 and A003, as QTL involved in flower color have been mapped on A002 and A003. Although the number of loci involved in flower color does not seem large, a simple genetic model could not be deduced. On the basis of our results, dominance relations between red and yellow could not be inferred. Some of the QTL were involved in more than one trait. For example, the QTL at E+ACCT/ M+CGCA-I165 on linkage group A002-3 was involved in L1 and L2, which suggests a pleiotropic effect of a single QTL. Similarly, on linkage group A003-9 two closely linked markers E+AATT/M+CGAT-222 and E+AATT/M+CGAT-248 each map a QTL involved in color traits $\mathrm{C} 1$ and $\mathrm{C} 3$ respectively, also suggesting the presence of a single QTL with pleiotropic effect. The presence of two different QTL with close linkage is not very likely in these cases, because of the highly related leaf and color phenotypes, respectively.

In this study, QTL for ornamental characters have been mapped on Alstroemeria linkage maps using AFLP markers mapped on nonintegrated parental linkage groups of A002 and A003. It was observed that many of the QTL did not reach high LOD values, or did not reach highly significant K-values in the Kruskal-Wallis test. It was also observed that QTL detected by interval mapping were not often confirmed by the Kruskal-Wallis test and vice versa. One of the obvious explanations is that our approach is not good. The phenotype is the joint result of the allele(s) derived from A002 and A003, and therefore, QTL should be mapped with the integrated map instead of the separate parental maps. Possibly some QTL would have reached much higher LOD scores when data from an integrated map were available, and possibly more QTL would have been detected. Although this is in principle true, unfortunately in practice this is not easily achieved. A large amount of codominant and multiallelic markers such as SSR or RFLP, resulting in a 1:1:1:1 segregation is required to obtain full classification of the offspring, and to obtain a good integrated map. In Alstroemeria, such markers have not been developed, and moreover, it will not be easy to develop such single locus markers in view of the limitations posed by the very large genome size of Alstroemeria. The signal detection threshold for RFLP is at picogram amounts of DNA on a Southern Blot, but before reaching such amounts of target se- quence, diluted in the huge genome, then probably the binding capacity of such a blot has been exceeded. The reproducibility of single locus PCR based markers such as SSR may be affected in large genome species. As a consequence the choice of AFLP over RFLP and SSR in this study was based on the high multiplex ratio, reproducibility and low development time/costs of AFLP.

Moreover, the development of SSR via the identification or enrichment of simple sequence repeat in DNA sequences is also hampered by the large genome.

The only markers that could be used for the integration of homologous A002 and A003 linkage groups were the $3: 1$ segregating AFLP markers. Maliepaard et al. (1997) have described the poor accuracy of the position of these 3:1 markers relative to the position of the 1:1 markers. The MapQTL ${ }^{\circledR}$ option for crosspollinators will use the information of the nearest flanking 1:1 markers to estimate the full genotypes at a certain position on the linkage group. In this way, information of several 1:1 and/or 3:1 markers are used to compensate for the missing information, and allow to reach the power of a 1:1:1:1 segregating marker for QTL analysis. We assume that ambiguities in marker order in the integrated map will have such a negative effect on these estimated genotypes at a certain map position, that the power of interval mapping and the Kruskal-Wallis test will be affected severely. In that case the gain of power by integration of the map is lost by the inaccuracies in marker order.

Another explanation for the poor significance of the QTL could be found in the (effective) population size. Although 134 offspring genotypes have been genotyped and phenotyped, this number has not always been accomplished. The amount of missing values in the AFLP data is $\approx 14 \%$, mainly caused by Sse8387I /MseI markers. The Sse8387I /MseI markers were applied on a subset of 90 genotypes only (one micro titre plate). For morphological/ornamental traits that were studied in the flower, a large number of missing values were caused by 49 genotypes that did not flower. However, the remaining 85 genotypes that did flower provided a population size that is not much lower than often seen in literature.

In many papers QTLs in $\mathrm{F}_{1}$ populations derived from non-inbreds are published where the majority of the QTL are just above the threshold. Grattapaglia et al. (1995) published QTL with LOD scores between 1.3 and 2.7 (one QTL at LOD $=5.8$ ) in a 122 offspring. Byrne et al. (1997) published leaf area QTL detected with the $F$ test at significance levels of $0.045<P<0.009$ using a 118 population size. King and Maliepaard et al. (2000) found in an offspring of 152 genotypes LOD scores up to 14.8 , but the majority of QTL had LOD values ranging between 3 and 4.5. From this perspective it seems that high LOD values are not easily reached in mapping populations derived from non-inbred parents. This could be based on the high number of possible allele combination resulting from heterozygosity in both the male and female parent. Up to four different alleles can be accommodated on a single locus in non-inbred mapping populations, where alleles of intermediate genotypic value will not add to the power of detection of such a QTL. The common existence of series of multiple alleles at QTL loci was discussed by Groover et al. (1994) in Eucalyptus and by Van Eck et al. (1994b) in potato. Although the LOD scores were not very high, we have not been overly greedy to mention QTLs of questionable significance. Two analytical approaches have been used in this paper: The Kruskal-Wallis test and interval mapping. It is generally assumed that the Kruskal-Wallis test is a conservative test, not easily resulting in false positives. Because the test was performed on 10 and 12 linkage groups of A002 and A003 respectively, a stringent significance level for the individual tests of $P=0.005$ was used in order to obtain an overall significance level of $\approx 0.05$. To control the significance level for interval mapping we used the permutation test (Churchill and Doerge, 1994), implemented in MapQTL ${ }^{\circledR}$. Due to the massive amount of calculation, however, Van Ooijen (1999) described an alternative method of getting the significance threshold without permutation test. However, current computer capacity allowed us to calculate significance thresholds for each trait, taking into account the quality of the map and the trait values. In this study significance LOD thresholds ranging between $\mathrm{LOD}=3.2-\mathrm{LOD}=4.9$ were obtained at the $99 \%$ confidence level. At this level we detected a total of 3 QTL on 3 linkage groups, and additionally 11 QTL on nine linkage groups at the $95 \%$ confidence level.

\section{Literature Cited}

Bennett, M.D., A.V. Cox, and I.J. Leitch. 1998. Angiosperm DNA $C$-values database. http:// www.rbgkew.org.uk/cval/database1.html.

Buitendijk, J.H., E.J. Boon, and M.S. Ramanna. 1997. Nuclear DNA content in twelve species of Alstroemeria $\mathrm{L}$. and some of their hybrids. Ann. Bot. 79:343-353.

Byrne, M., J.C. Murrell, J.V. Owen, P. Kriedemann, E.R. Williams, and G.F. Moran 1997. Identification and mode of action of quantitative trait loci affecting seedling height and leaf area in Eucalyptus nitens. Theor. Appl. Genet. 94:674681

Churchill, G.A., and R.W. Doerge. 1994. Empirical threshold values for quantitative trait mapping. Genetics 138:963-971.

Debener, T. and L. Mattiesch. 1999. Construction of a genetic linkage map for roses using RAPD and AFLP markers. Theor. Appl. Genet. 99:891-899.

Gentzbittel, L., E. Mestries, S. Mouzeyar, F. Mazeyrat, S. Badaoui, F. Vear,D.T. de Labrouhe, and P. Nicolas. 1999. A composite map of expressed sequences and phenotypic traits of the sunflower (Helianthus annuus L.) genome. Theor. Appl. Genet. 99:218-234.

Grandillo, S., H.M. Ku, S.D. Tanksley. 1999. Identifying the loci responsible for natural variation in fruit size and shape in tomato. Theor. Appl. Genet. 99:978-987.

Grattapaglia, D., F.L. Bertolucci, R.R. Sederoff. 1995. Genetic mapping of QTLs controlling vegetative propagation in Eucalyptus grandis and E. urophylla using a pseudo-testcross strategy and RAPD markers. Theor. Appl. Genet. 90:933-947. 
Groover, A., M. Devey, T. Fiddler, J.Lee, R. Megraw, T. Mitchel-Olds, B. Sherman, S. Vujcic, C. Williams, and D. Neale. 1994. Identification of quantitative trait loci influencing wood specific gravity in an outbred pedigree of loblolly pine. Genetics 138:1293-1300.

Han, T.H., H.J. Van Eck, M.J. De Jeu, and E Jacobsen. 1999. Optimization of AFLP fingerprinting of organisms with a large genome size: A study on Alstroemeria spp. Theor. Appl. Genet. 98:465-471.

Han, T.H., M.J. De Jeu, H. Van Eck, and E. Jacobsen. 2000. Genetic diversity of Chilean and Brazilian Alstroemeria species assessed by AFLP analysis. Heredity 84:564-569.

Han, T.H., H.J. Van Eck, M.J. De Jeu, and E. Jacobsen. 2001. Linkage map construction of Alstroemeria aurea by AFLP markers. Euphytica (In Press.)

Jiang, C., R.J. Wright, S.S. Woo, T.A. DelMonte, and A.H. Paterson. 2000. QTL analysis of leaf morphology in tetraploid Gossypium (cotton). Theor. Appl. Genet. 100:409-418.

Kato, K., H. Miura, and S. Sawada. 1999. QTL mapping of genes controlling ear emergence time and plant height on chromosome $5 \mathrm{~A}$ of wheat. Theor. Appl. Genet. 98:472-477.

King, G.J., C. Maliepaard, J.R. Lynn, F.H. Alston, C.E. Durel, K.M. Evans, B. Griffon, F. Laurens, A.G. Manganaris, E. Schrevens, and S. Tartarini. 2000. Quantitative genetic analysis and com- parison of physical and sensory descriptors relating to fruit flesh firmness in apple (Malus pumila Mill). Theor. Appl. Genet. 100:10741084.

Kruglyak, L. and E.S. Lander. 1995. A nonparametric approach for mapping quantitative trait loci. Genetics 139:1421-1428

Lander, E.S. and D. Botstein. 1989. Mapping Mendelian factors underlying quantitative traits using RFLP linkage maps. Genetics 121:185-199.

Maliepaard, C., J. Jansen, and J.W. Van Ooijen. 1997. Linkage analysis in a full-sib family of an outbreeding plant species: overview and consequences for applications. Genet. Res. 70:237250 .

Union internationale pour la protection des obtentions vegetales (UPOV). 1987. Guidelines for the conduct of tests for distinctness, homogeneity and stability. TG/29/6 (revision of TG/29/3) http://www.upov.org.

Van Heusden, A.W., J.W. Van Ooijen, R. Vrielinkvan Ginkel, W.H.J. Verbeek, W.A. Wietsma, and C. Kik. 2000. A genetic ma0-p of an interspecific cross in Allium based on amplified fragment length polymorphism (AFLP ${ }^{\mathrm{TM}}$ ) markers. Theor. Appl. Genet. 100:118-126.

Van Eck H.J., J.M.E. Jacobs, J. Van Dijk, W.J. Stiekema, and E. Jacobsen. 1993. Identification and mapping of three flower colour loci of potato (S. tuberosum L.) by RFLP analysis. Theor. Appl. Genet. 86:295-300.
Van Eck H.J., J.M.E. Jacobs, P.M.M.M. Van den Berg, W.J. Stiekema, E. Jacobsen. 1994a. The inheritance of anthocyanin pigmentation in potato (Solanum tuberosum L.) and mapping of tuber skin colour loci using RFLPs. Heredity 73:410-421.

Van Eck H.J., J.M. Jacobs, P. Stam, J. Ton, W.J. Stiekema, and E. Jacobsen. 1994b. Multiple alleles for tuber shape in diploid potato detected by qualitative and quantitative genetic analysis using RFLPs. Genetics 137:303-309.

Van Ooijen J.W. 1999. LOD significance thresholds for QTL analysis in experimental populations of diploid species. Heredity 83:613624.

Van Ooijen J.W. 2000. MapQTL ${ }^{\circledR}$ Version 4.0: User friendly power in QTL mapping. Plant Research International, http://www.plant.wageningenur.nl/products/mapping/MapQTL/mqintro.htm.

Voorrips, R.E. 2000. MapChart version 1.4: Windows software for the graphical presentation of linkage maps and QTLs. Plant Res. Intl., Wageningen.

Zhu H., L. Gilchrist, P. Hayes, A. Kleinhofs, D. Kudrna, Z. Liu, L. Prom, B. Steffenson, T. Toojinda, and H. Vivar. 1999. Does function follow form? Principal QTL for Fusarium head blight (FHB) resistance are coincident with QTL for inflorescence traits and plant height in a doubled-haploid population of barley. Theor. Appl. Genet. 99:1221-1232. 